\title{
Sistema reproductivo de Trichloris (Poaceae, Chloridoideae, Chlorideae)
}

\author{
HUGO FRANCISCO GUTIÉRREZ1', GERALDINA ALICIA RICHARD 1, 2, MARÍA CAROLINA \\ CERINO ${ }^{1,2}$ y JOSÉ FRANCISCO PENSIERO ${ }^{1,2}$
}

\begin{abstract}
Summary: Reproductive system of Trichloris (Poaceae, Chloridoideae, Chlorideae). Trichloris crinita and T. pluriflora are important native forage species with the potential to be domesticated and introduced to cultivation, but there is scarce basic information on their reproductive biology. The aim of this work was to determine the reproductive system of both plant species in populations coming from north-central Argentina. The following was proposed: 1) determine the reproductive system through the fruit/flower ratio under controlled pollination, 2) quantify the weight of the fruits produced by population under different treatments and 3) confirm or discard the presence of apomixis through embryological observations. In $T$. crinita, the average fruit/flower ratio was $92.4 \pm 6.6 \%$, while in $T$. pluriflora, it was $70.7 \pm 9.9 \%$. These results are consistent with self-compatible species, preferably self-pollinated ones. Embryo sac development indicates sexual origin of seeds in both species. Significant differences in the average weight of one hundred fruits among populations and treatments, in both species, were found. The fruits were lighter in self-pollination. The information presented is a contribution to the tasks of selection and introduction to the culture of both species as well as to the conservation and regeneration of the collected germplasm.
\end{abstract}

Key words: Trichloris, Reproductive success, Self-pollination, Fruit weight.

Resumen: Trichloris crinita y T. pluriflora constituyen importantes forrajeras nativas con potencialidades de ser domesticadas e introducidas al cultivo, pero es escasa la información básica sobre su biología reproductiva. El objetivo de este trabajo fue determinar el sistema reproductivo de ambas especies en poblaciones provenientes del centro-norte de Argentina. Se propuso: 1) establecer el sistema reproductivo a través de la relación fruto/flor en polinizaciones controladas, 2) cuantificar el peso de los frutos producidos, por población, bajo diferentes tratamientos y, 3) confirmar o descartar la presencia de apomixis a través de observaciones embriológicas. En T. crinita, la relación fruto/flor promedio fue de $92,4 \pm 6,6 \%$, mientras que en $T$. pluriflora, fue de 70,7 $\pm 9,9 \%$. Estos resultados se corresponden con especies autocompatibles, preferentemente autógamas. El desarrollo del saco embrionario indica origen sexual de las semillas en ambas especies. Se hallaron diferencias significativas en el peso promedio de cien cariopsis entre poblaciones y entre tratamientos, en ambas especies. Los frutos fueron más livianos en autopolinización. La información que se presenta es un aporte a las tareas de selección e introducción al cultivo como también a la conservación y regeneración del germoplasma recolectado.

Palabras clave: Trichloris, Éxito reproductivo, Autopolinización, Peso de frutos.

\section{INTRODUCCIÓN}

En Argentina, el avance de la agricultura desplazó las actividades ganaderas hacia ambientes

\footnotetext{
${ }^{1}$ Cátedra de Botánica Sistemática Agronómica, Facultad de Ciencias Agrarias, Universidad Nacional del Litoral, RP Luis Kreder 2805, 3080HOF Esperanza, Argentina.

${ }^{2}$ Consejo Nacional de Investigaciones Científicas y Técnicas, Buenos Aires, Argentina.
}

marginales, algunos de los cuales presentan serias limitaciones como anegamiento, salinidad o sequía. Esto motivó la búsqueda de nuevos recursos forrajeros, adaptados a estos ambientes más restrictivos. En este sentido, las especies del género Trichloris podrían constituir valiosos recursos para incluir en planes de domesticación de forrajeras. La flora de Argentina se caracteriza por la presencia de numerosas gramíneas nativas con cualidades forrajeras para ser introducidas al cultivo (Covas, 1978, Zuloaga et al., 1994). Entre ellas se destacan 
las especies de Trichloris E. Fourn. ex Benth. (Poaceae, Chlorideae). El género es exclusivamente americano y está representado por dos especies: T. crinita (Lag.) Parodi y T. pluriflora E. Fourn. Ambos taxones son perennes, $\mathrm{C} 4$ y se distribuyen a través de toda América, con excepción de Canadá (Peterson, 2001). Se encuentran preferentemente en ambientes áridos y semiáridos, pudiendo habitar además, en sitios subhúmedos y húmedos (Ragonese, 1956, Covas, 1978, Cavagnaro, 1988, Cabrera, 1994, Zabala et al., 2011).

Trichloris crinita se destaca por su producción forrajera, ofreciendo forraje palatable y de buena calidad (Bragadín, 1959, Wainstein \& González, 1971, Cerqueira et al., 2004). Esta especie es tolerante a la defoliación (Cavagnaro \& Dalmasso, 1983), a la sequía (Greco \& Cavagnaro, 2003) y a la salinidad (Aronson, 1989, Lloyd-Reilley \& Kadin, 2002) y su presencia en los pastizales es indicadora de buena condición forrajera (Kunst et al., 2007).

Trichloris pluriflora presenta una oferta forrajera abundante (Kunst et al., 1995), de aceptable digestibilidad y calidad (Kunst et al., 2007), siendo muy apetecida por el ganado (Díaz, 1965a, b, Kunst, et al., 1986, Kunst et al., 1987, Kunst et al., 1995). Kunst et al. (2007) la consideran indicadora de buena condición en los pastizales, siendo abundante en sitios con alta disponibilidad de luz de los bosques chaqueños (Morello \& Saravia Toledo, 1959, Bordón, 1981).

En relación al sistema reproductivo y la producción de frutos, en T. crinita, Greco \& Cavagnaro (2005) y Cavagnaro et al. (2006) sugirieron la presencia de apomixis o autogamia obligada, al no hallar variaciones fenotípicas en las descendencias de plantas madre luego de cuatro generaciones. Para T. pluriflora se desconoce su sistema reproductivo. La falta de información referida a la biología reproductiva de ambas especies constituye una barrera si se pretende su domesticación e introducción al cultivo (Fehr, 1987). El presente trabajo se propone: 1) establecer el sistema reproductivo a través de polinizaciones controladas, 2) determinar el peso de los frutos producidos y, 3) confirmar o descartar la presencia de apomixis a través del estudio del desarrollo de los sacos embrionarios.

\section{Materiales y Métodos}

\section{Descripción de las especies}

El género Trichloris tiene inflorescencias formada por racimos de espiguillas, reunidos en un fascículo en la porción apical de las cañas; además la lemma inferior de cada espiguilla es 3-aristada, siendo la longitud de las aristas centrales de 8-12 mm, y la de las laterales de 0,5-12 mm (Nicora \& Rúgolo, 1987). Trichloris crinita es una hierba perenne, hasta $1 \mathrm{~m}$ de altura, cespitosa, rizomatoso-estolonífera, de cañas generalmente simples, erectas o geniculadas. Su inflorescencia está formada por 5-25 racimos espiciformes de 5-15 cm de longitud y sus espiguillas presentan 1 antecio inferior perfecto y fértil y 1-2 superiores, neutros y estériles. Trichloris pluriflora es una hierba perenne, hasta $1,5 \mathrm{~m}$ de altura, cespitosa, de cañas generalmente simples y erectas, con porte más robusto que $T$. crinita. Su inflorescencia está formada por 7-20 racimos espiciformes de $7-20 \mathrm{~cm}$ de longitud y sus espiguillas presentan 2-4 antecios inferiores perfectos y fértiles y 1-3 superiores, neutros y estériles, que son más pequeños hacia el ápice (Nicora \& Rúgolo, 1987).

\section{Material vegetal}

Se cosecharon cariopsis de 15-20 individuos por población, los que se dispusieron en el mismo sobre manteniendo la individualidad de la procedencia. En total se colectaron 8 poblaciones de Trichloris (4 de T. crinita y 4 de T. pluriflora), procedentes de 6 provincias de Argentina (Tabla 1).

Una muestra representativa de alrededor de 250 frutos de cada población fue sembrada en bandejas plásticas sobre sustrato de arena; luego las plántulas fueron llevadas a campo y mantenidas en el Jardín Experimental de la Facultad de Ciencias Agrarias de la Universidad Nacional del Litoral, Esperanza, provincia de Santa Fe $\left(31^{\circ} 27^{\prime} \mathrm{S}, 60^{\circ} 56^{\prime} \mathrm{O}\right)$. En el ensayo, las plantas fueron ubicadas en un diseño completamente aleatorizado con 6 repeticiones. Cada repetición estuvo integrada por 5 plantas por población, distanciadas a $0,50 \mathrm{~m}$ entre plantas y filas. Durante el ensayo se realizaron riegos periódicos y desmalezados a mano. En el mes de diciembre se realizó la cosecha de frutos de todas las plantas. 


\section{H. F. Gutiérrez et al. - Sistema reproductivo de Trichloris}

Tabla 1. Procedencia de las poblaciones estudiadas $\left({ }^{*}\right)$

\begin{tabular}{|c|c|c|c|c|c|}
\hline \multirow{2}{*}{ Especie } & \multirow{2}{*}{$\mathbf{N}^{\circ}$} & \multirow{2}{*}{ Provincia } & \multirow{2}{*}{ Ubicación } & \multicolumn{2}{|c|}{ Coordenadas GPS } \\
\hline & & & & Sur & Oeste \\
\hline \multirow{4}{*}{ T. crinita } & 1 & San Luis & INTA San Luis & \multicolumn{2}{|c|}{ Material seleccionado } \\
\hline & 2 & Santiago del Estero & Dpto. Robles. Ruta 34, mojón 697,5 & $27^{\circ} 51^{\prime} 23,95^{\prime \prime}$ & $63^{\circ} 59^{\prime} 47,1^{\prime \prime}$ \\
\hline & 3 & Formosa & $\begin{array}{l}\text { Dpto. Formosa. Ruta } 81 \text {, } \\
\text { próximo a Corralito. }\end{array}$ & $25^{\circ} 41^{\prime} 14,3^{\prime \prime}$ & $59^{\circ} 03^{\prime} 48,0^{\prime \prime}$ \\
\hline & 4 & Córdoba & $\begin{array}{l}\text { Dpto. Tulumba. Salinas Grandes, } \\
\text { próximo a Lucio V. Mancilla }\end{array}$ & $29^{\circ} 52^{\prime} 38,3^{\prime \prime}$ & $64^{\circ} 39^{\prime} 56,0^{\prime \prime}$ \\
\hline \multirow{4}{*}{ T. pluriflora } & 1 & Córdoba & $\begin{array}{l}\text { Dpto. Punilla. Ruta } 17,1,6 \\
\text { km al este de la Ruta } 38 .\end{array}$ & $30^{\circ} 47^{\prime} 56,8^{\prime \prime}$ & $64^{\circ} 31^{\prime} 29,2^{\prime \prime}$ \\
\hline & 2 & Salta & $\begin{array}{l}\text { Dpto. Metán. Ruta } 5 \text {, } \\
\text { camino a Lumbrera }\end{array}$ & $25^{\circ} 13^{\prime} 0,9^{\prime \prime}$ & $64^{\circ} 55^{\prime} 46,3^{\prime \prime}$ \\
\hline & 3 & Salta & $\begin{array}{l}\text { Dpto. Orán. Ruta 5, de La } \\
\text { Estrella a Chaguaral. }\end{array}$ & $24^{\circ} 03^{\prime} 17,0^{\prime \prime}$ & $64^{\circ} 00^{\prime} 15,7^{\prime \prime}$ \\
\hline & 4 & Jujuy & Dpto. El Carmen. Ruta 9 (en banquina) & $24^{\circ} 32^{\prime} 15,5^{\prime \prime}$ & $65^{\circ} 04^{\prime} 50,7^{\prime \prime}$ \\
\hline
\end{tabular}

(*) Un ejemplar herborizado de cada procedencia se halla depositado en el Herbario SF. Los números de colección son: T. crinita, Pensiero et al. 7078 (2), 6917 (3) y 6975 (4). T. pluriflora, Pensiero et al. 6962 (1), 7000 (2), 7063 (3) y 7011 (4).

\section{Determinación del éxito reproductivo}

Se calculó la relación entre el número de frutos y el de flores de cada planta (relación fr/fl) y se registró el peso de 100 cariopsis obtenido en cada tratamiento. Los tratamientos evaluados fueron:

1) Polinización libre (testigo): las plantas permanecieron en polinización libre hasta el final de su floración. Una vez finalizada la misma, las inflorescencias fueron ensobradas con el fin de evitar pérdidas por desgrane. En total se evaluaron 120 plantas experimentales (15 plantas por población y por especie).

2) Autopolinización forzada: una inflorescencia completa de cada una de las plantas experimentales del tratamiento 1 fue ensobrada (utilizando sobres de papel parafinado de 20 $\mathrm{cm}$ de longitud x $4 \mathrm{~cm}$ de ancho) en estadios tempranos (antes del inicio de la floración), cuando las inflorescencias se encontraban aún casi totalmente envueltas por la vaina foliar. Así fueron mantenidas hasta la madurez del grano, permitiendo la autopolinización y la geitonogamia. En total se evaluaron 120 plantas experimentales (15 plantas por población y por especie).
3) Aislamiento: en el mismo momento de los tratamientos 1 y 2 , se eligieron al azar 3 plantas experimentales por población, las que se plantaron individualmente en macetas plásticas de 5 litros. Dichas plantas se mantuvieron aisladas de otros individuos de la misma especie por distancias superiores a $600 \mathrm{~m}$, y así permanecieron (al aire libre y sin ensobrar) hasta la madurez del grano. Si bien en los tratamientos 2) y 3) los frutos formados provienen de autopolinización (o apomixis), se diferencian en que en el tratamiento 3 , no existe el factor "sobre" (ensobrado) que podría inducir un cambio en las condiciones microambientales que rodean a la inflorescencia y afectar su producción.

La determinación del éxito reproductivo se realizó bajo microscopio estereoscópico, contando el número de antecios que presentaron cariopsis, de acuerdo a la expresión: Fecundidad $(\%)=$ (número de cariopsis formados/número de antecios) * 100. En gramíneas, el gineceo es 1-ovulado, por lo tanto la expresión propuesta por Wiens et al. (1987) queda reducida a la relación frutos/flores ya que la proporción semilla/óvulo es igual a 1. Este cociente fue calculado para toda la población en cada condición. 


\section{Peso de cien frutos}

Para analizar la variable "peso de 100 frutos" (Johnston et al., 2003), se extrajo una muestra de cariopsis de 10 plantas experimentales por población para los tratamientos autopolinización y polinización libre y de 3 plantas por población para el tratamiento de aislamiento. Se mezclaron los frutos de diferentes plantas de cada tratamiento, conformando un pool. De cada pool se pesaron 8 muestras de 50 cariopsis por población y por especie (para autopolinización y polinización libre) y 3 muestras de 50 cariopsis por población y por especie (para asilamiento). Los valores obtenidos se expresaron como peso (en gramos) de 100 frutos.

\section{Tratamiento estadístico}

En cada especie se realizó el análisis de la varianza (ANVA) para la variable relación fr/ fl, con el objetivo de analizar el efecto del factor población (4 niveles), tratamiento de polinización (3 niveles) y su interacción sobre el éxito reproductivo. En Trichloris crinita los valores de fr/fl debieron ser transformados al arcoseno (Gutiérrez et al., 2006) para satisfacer el supuesto de normalidad de datos del ANVA. En la comparación de medias se utilizó el Test de Tukey $(p<0.05)$. Los datos fueron analizados mediante el paquete estadístico Infostat (2014). Idéntico procedimiento se realizó para la variable peso de 100 frutos.

\section{Estudios embriológicos}

Con la finalidad de establecer la presencia o ausencia de apomixis, se realizaron observaciones en el desarrollo de los sacos embrionarios en ambas especies, principalmente en lo que se refiere a la megasporogénesis y megagametogénesis. Se procuró constatar la ocurrencia de meiosis y la formación de una célula madre de la megáspora reducida a partir de la cual se origine un saco embrionario. Para ello se fijaron inflorescencias en 3 estadios de floración: a) comienzo de emergencia (se percibe un 10\% de la inflorescencia exerta), b) fin de emergencia (los racimos se encuentran totalmente emergidos) y c) mitad de emergencia (situación intermedia entre a y b). Las inflorescencias fueron fijadas en formol, ácido acético y alcohol (Ruzin, 1999) durante
24 horas y almacenadas en etanol $70 \%$ a $4{ }^{\circ} \mathrm{C}$. Este material se trató posteriormente con ácido fluorhídrico $20 \%$ durante 48 horas para disolver las sílices. Una vez libre de sílice, se procedió a su deshidratación e inclusión en parafina mediante una serie de alcohol-xilol-parafina (D'Ambrogio, 1986). Se cortaron secciones de 7-9 $\mu \mathrm{m}$ de espesor con micrótomo rotatorio y fueron teñidas con safranina y fast-green (D’Ambrogio, 1986).

\section{Resultados}

\section{Éxito reproductivo}

Para la variable fr/fl no se hallaron diferencias significativas entre bloques, ni en la interacción "tratamiento x población" para cada una de las especies evaluadas. En Trichloris crinita la fecundidad media fue de $92,4 \pm 6,6 \%$, sin que se registren diferencias significativas entre poblaciones $(\mathrm{F}=1,46 ; p=0,23)$, ni entre tratamientos $(\mathrm{F}=2,30 ; p=0,10)$ (Figura 1). En T. pluriflora la fecundidad media fue de $70,7 \pm$ $9,9 \%$, hallándose diferencias significativas entre poblaciones $(\mathrm{F}=3,17 ; p=0,03)$, pero no entre tratamientos $(\mathrm{F}=1,89 ; p=0,16)$ (Figura 2).

\section{Peso de 100 frutos}

Para la variable peso de 100 cariopsis, la interacción "tratamiento x población" no tuvo efecto significativo en $T$. crinita $(\mathrm{F}=0,66 ; \mathrm{p}=$ $0,68)$, pero si en $T$. pluriflora $(\mathrm{F}=8,38 ; p<$ $0,0001)$. En $T$. crinita el peso promedio fue $0,181 \pm 0,043 \mathrm{~g}$, con diferencias significativas entre poblaciones $(\mathrm{F}=83,9 ; p<0,0001)$ y entre tratamientos $(\mathrm{F}=6,09 ; p=0,004)$ (Figura 3). A nivel de especie ( $\sin$ considerar las distintas poblaciones) los frutos de T. crinita obtenidos en polinización libre y en plantas aisladas resultaron más pesados que los obtenidos bajo autopolinización forzada. ( $\mathrm{F}=9,63 ; p=0,0005)$. En T. pluriflora el peso promedio fue $0,165 \pm$ $0,044 \mathrm{~g}$, hallándose diferencias significativas entre poblaciones $(\mathrm{F}=6,54 ; p=0,0006)$ y entre tratamientos $(\mathrm{F}=16,03 ; p<0,0001)$ (Figura 4). A nivel de especie, los frutos de $T$. pluriflora que resultaron más pesados correspondieron a los tratamientos de polinización libre y plantas aisladas $(\mathrm{F}=7,92 ; p=0,0008)$. 


\section{H. F. Gutiérrez et al. - Sistema reproductivo de Trichloris}

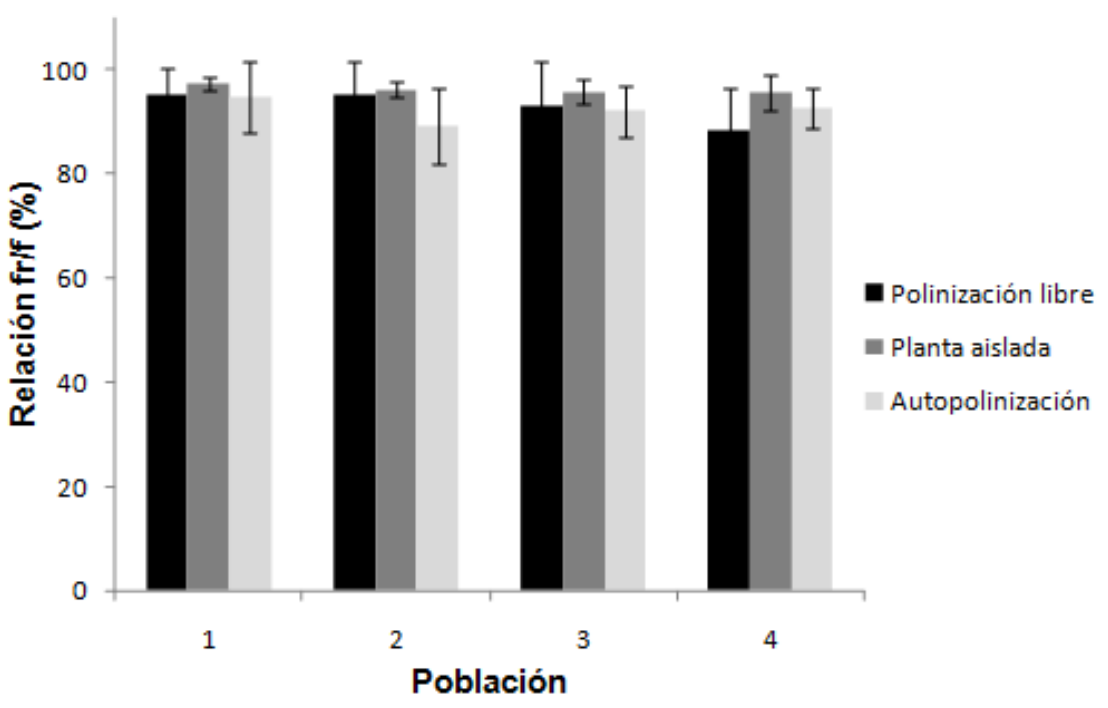

Fig. 1. Relación fruto/flor en 4 poblaciones de Trichloris crinita sometidas a 3 tratamientos de polinización.

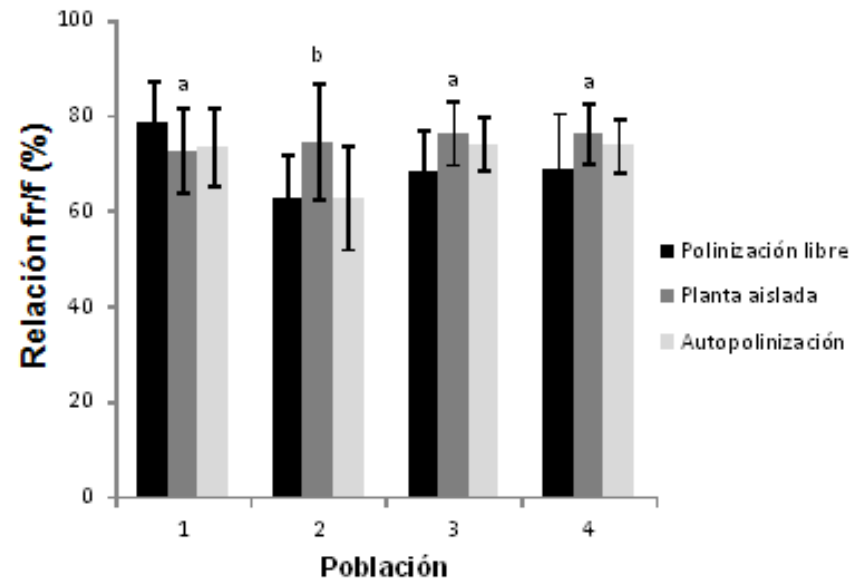

Fig. 2. Relación fruto/flor en 4 poblaciones de Trichloris pluriflora sometidas a 3 tratamientos de polinización.

\section{Embriología}

El desarrollo de los sacos embrionarios se pudo observar con claridad solamente en las inflorescencias fijadas en el estadio "a". En los estadios posteriores no se visualizaron sacos embrionarios, dado el estado avanzado de desarrollo de la semilla. En todas las espiguillas evaluadas de ambas especies de Trichloris se constató la presencia de un solo megagametofito por óvulo y no se detectaron células iniciales apospóricas. El desarrollo del gametofito femenino correspondió al tipo Polygonum, constatándose la ocurrencia de meiosis y la formación de una célula madre de la megáspora reducida a partir de la cual se originó un saco embrionario constituido por dos sinérgidas, una célula huevo, dos grandes núcleos polares y un racimo de células antípodas (Fig. 5 y 6). 
Bol. Soc. Argent. Bot. 51 (1) 2016

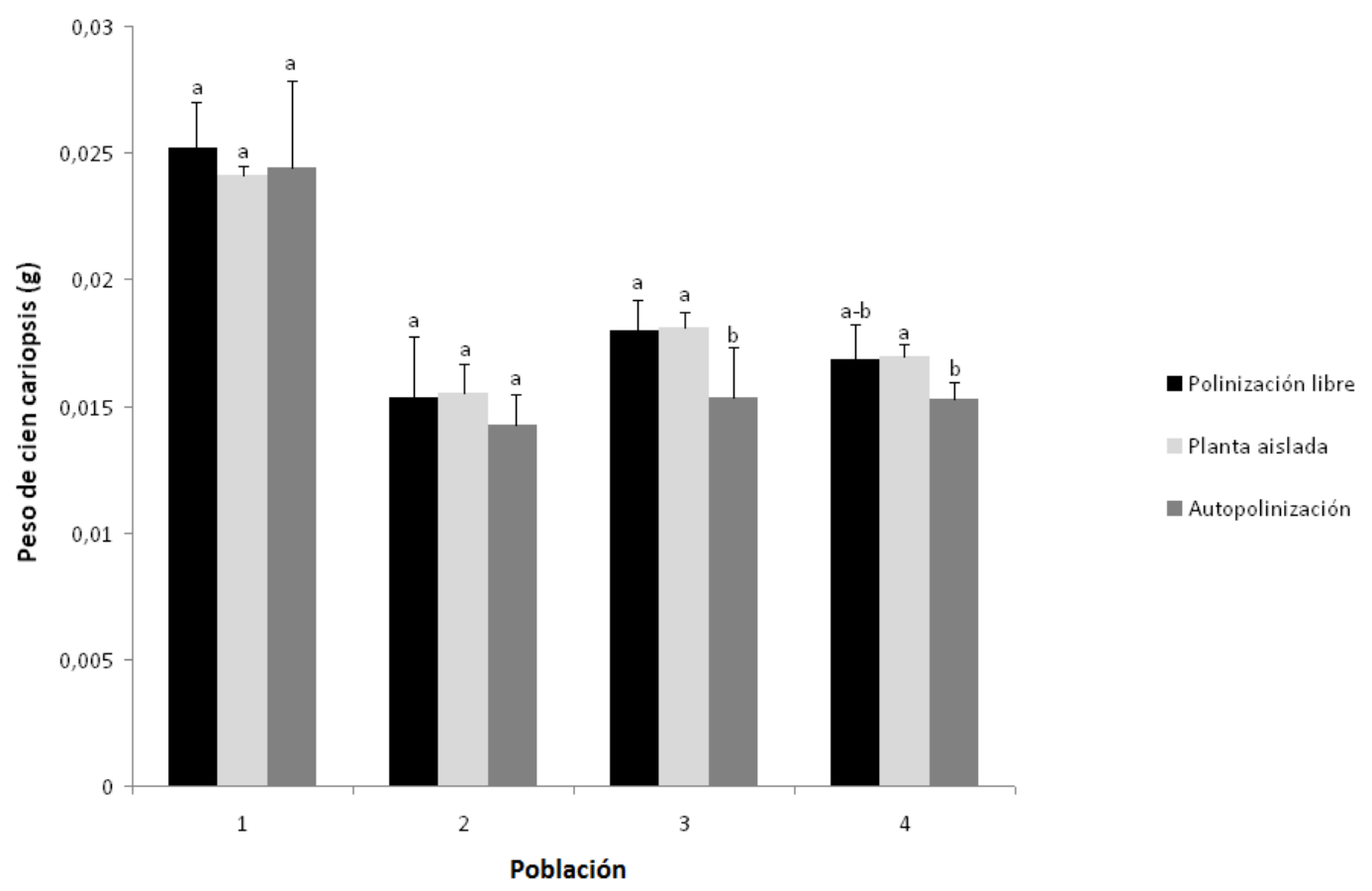

Fig. 3. Peso promedio de cien cariopsis en 4 poblaciones de Trichloris crinita en polinización libre, plantas reproductivamente aisladas y en autopolinización forzada (ensobrado de inflorescencia).

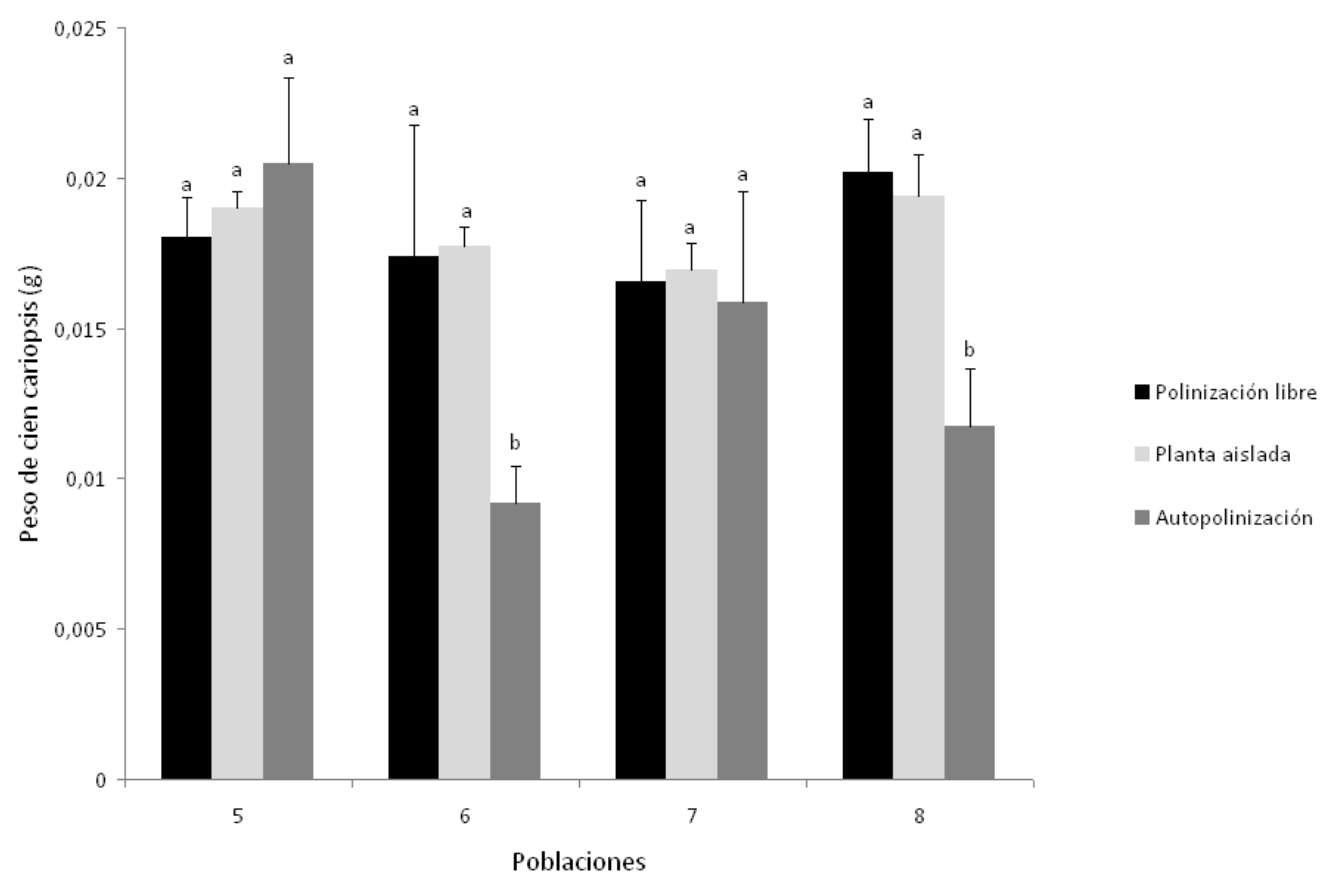

Fig. 4. Peso promedio de cien cariopsis en 4 poblaciones de Trichloris pluriflora en polinización libre, plantas reproductivamente aisladas y en autopolinización forzada (ensobrado de inflorescencia). 


\section{H. F. Gutiérrez et al. - Sistema reproductivo de Trichloris}
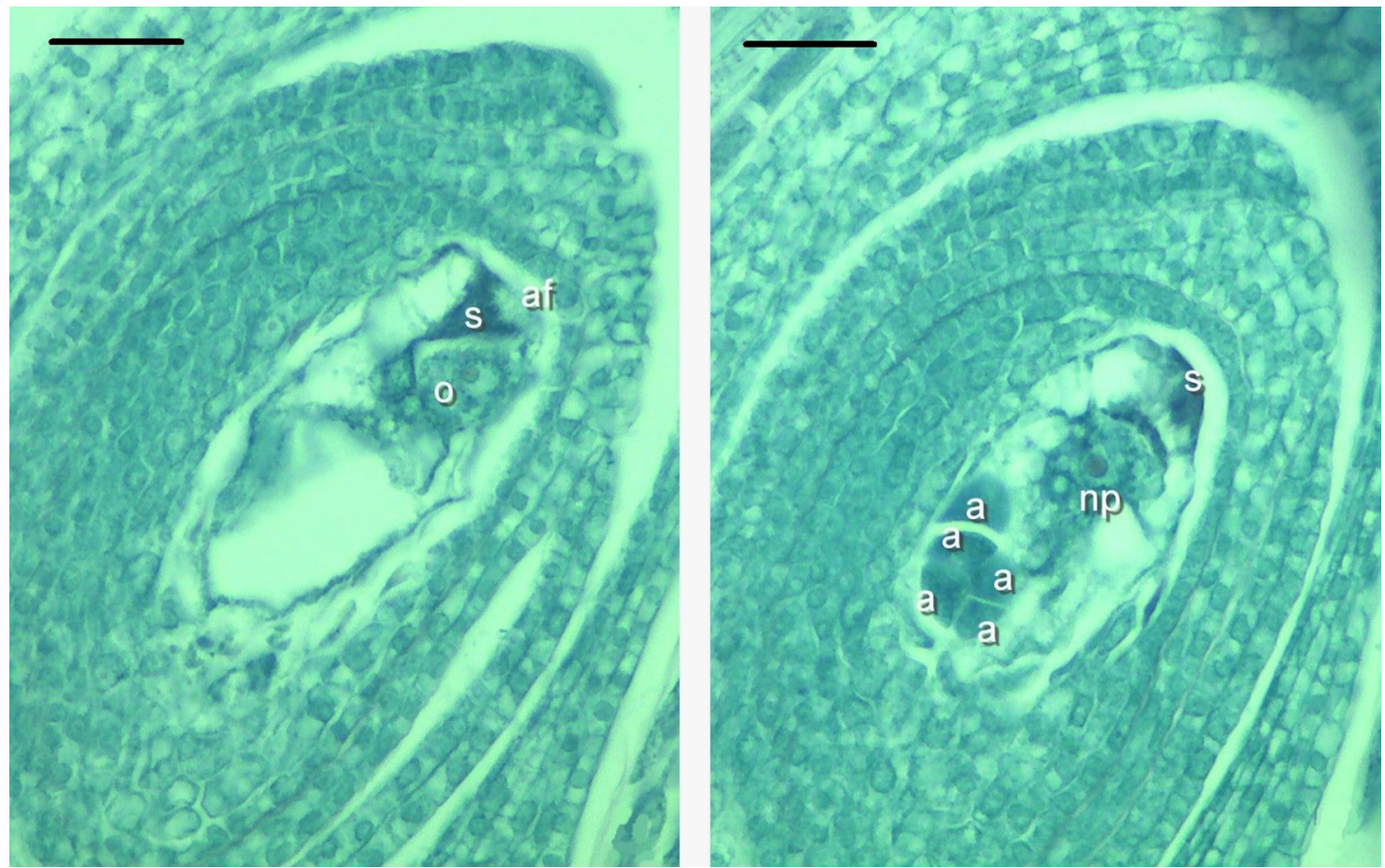

Fig. 5. Megagametofito maduro de Trichloris crinita. Ref. o: oósfera, s: sinérgida, af: aparato filar, np: núcleo polar, a: antípodas. Longitud de la barra $=10 \mathrm{um}$.

\section{Discusión}

Los resultados obtenidos en este trabajo indican que ambas especies de Trichloris se reproducen sexualmente y que el sistema reproductivo es autógamo.

Considerando a la relación fr/fl como un estimador indirecto del sistema reproductivo se pudo comprobar que, en las poblaciones de Trichloris evaluadas, el origen del polen (propio o de otra planta) no afectó la formación de frutos. Los elevados valores de fr/fl obtenidos confirman la existencia de autocompatibilidad en ambas especies, como fue inicialmente sugerido por Greco \& Cavagnaro (2005) y Cavagnaro et al. (2006) al evaluar progenies en $T$. crinita.

La fecundidad promedio superó, en todas las poblaciones, el $70 \%$, lo que indica que la producción de frutos de ambas especies no sería un factor limitante para su introducción a cultivo. La elevada relación fr/fl resulta alentadora por tratarse de forrajeras nativas aún no sometidas a un proceso de domesticación. A nivel poblacional, la producción de frutos de $T$. crinita fue superior a la de $T$. pluriflora. Esta diferencia podría estar dada por la cantidad de antecios fructíferos en las espiguillas de ambas especies. Trichloris crinita tiene un solo antecio inferior perfecto y potencialmente fructífero, mientras que en T. pluriflora la cantidad de antecios perfectos y potencialmente fructíferos varía de 2 a 4 . Por ello, en esta última especie la relación fr/fl presentó valores menores que $T$. crinita. En Poáceas, los antecios perfectos no fructíferos contribuyen al fitness masculino al actuar como donantes de polen, de acuerdo con la teoría de asignación sexual (Charnov \& Bull, 1986).

Dado que la producción de frutos no varió significativamente según el origen del polen (propio o ajeno), se descarta la xenogamia como sistema reproductivo predominante en ambas 
Bol. Soc. Argent. Bot. 51 (1) 2016
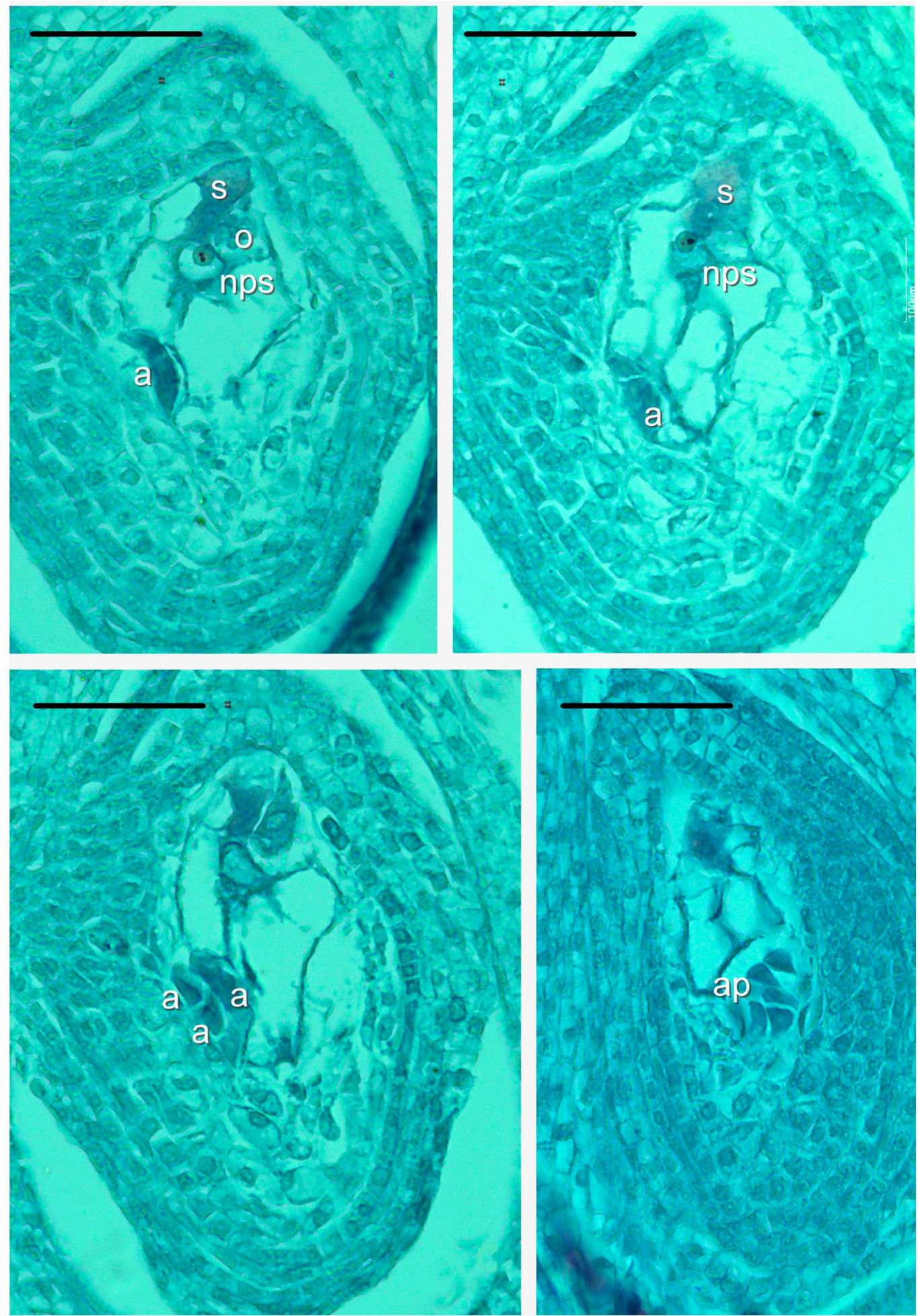

Fig. 6. Megagametofito maduro de Trichloris pluriflora. Ref. o: oósfera, s: sinérgida, nps: núcleos polares, a: antípodas. Longitud de la barra $=10 \mathrm{um}$. 


\section{H. F. Gutiérrez et al. - Sistema reproductivo de Trichloris}

especies. Cuando experimentalmente se redujo la transferencia de polen (al ensobrar inflorescencias $\mathrm{y}$ al evaluar la producción de frutos en plantas reproductivamente aisladas), la relación $\mathrm{fr} / \mathrm{fl}$ obtenida no varió en forma significativa. Esto confirma que el movimiento del polen en una misma planta (autopolinización y/o geitonogamia) fue suficiente para producir frutos. Por otra parte, el sobre utilizado para cubrir las inflorescencias no afectó la producción de frutos (autopolinización vs. polinización libre). Resultados similares al utilizar sobres fueron obtenidos en otras especies de gramíneas nativas de Setaria (Pensiero et al., 2005), Bromus (Gutiérrez et al., 2006) y Sporobolus (Aracne, 2010).

En un estudio preliminar, basado en el análisis de caracteres morfológicos de progenies provenientes de distintas accesiones, se sugirió que $T$. crinita podía presentar reproducción agámica a través de apomixis, o bien sexual con elevadas tasas de autogamia (Greco y Cavagnaro, 2005, Cavagnaro et al. 2006). Por su parte Kozub (2011) obtuvo resultados consistentes con la hipótesis de apomixis como método reproductivo para esta especie. Sin embargo en este trabajo se observó que las dos especies estudiadas presentan gametofitos femeninos del tipo Polygonum (Richard et al., inédito), típico en gramíneas con sistemas de reproducción sexual (Anton \& Cocucci 1984, Johri et al. 1992). En gramíneas apomícticas, Quero-Carrillo et al. (2010) señalaron como irregularidades características tanto anormalidades en la megasporogénesis como la presencia de células iniciales apospóricas. Estas irregularidades no fueron detectadas en ambas especies lo que nos permite sugerir el origen sexual de los frutos para el género Trichloris. La falta de segregación fenotípica hallada en progenies de T. crinita (Greco \& Cavagnaro, 2005, Cavagnaro et al., 2006) puede deberse entonces a una elevada tasa de autogamia.

Los valores de fructificación obtenidos y la ausencia de apomixis permiten postular que el sistema reproductivo en las especies de Trichloris es preferentemente autógamo. En general, la autogamia puede estar asociada con flores cleistógamas, flores casmógamas o con una combinación de ambas (Campbell et al., 1983). Si bien no se ha estudiado detalladamente la fenología floral, en este ensayo no se detectaron flores cleistógamas durante el ensobrado de las inflorescencias ni al determinar la relación fr/fl de los antecios. Diversos autores han señalado numerosos caracteres morfo-funcionales asociados a las especies autógamas. Entre estos se destacan: a) tamaño reducido de las estructuras florales (Goodwillie et al., 2010, Kalisz et al., 2012), b) elevada superposición temporal de las funciones masculina y femenina (Delesalle et al., 2008, Mazer et al., 2009), c) ciclos florales de corta vida (Runions \& Geber, 2000, Mazer et al., 2010), d) estructuras masculinas y femeninas próximas entre sí (Webb \& Lloyd, 1986, Herlihy \& Eckert, 2007) y, e) baja relación polen/óvulo (Cruden, 1977). Muchos de estos caracteres están presentes en las especies de Trichloris, reforzando su condición de autógamas. En este contexto, las flores casmógamas aportarían la fracción de variabilidad que explicaría las diferencias poblacionales detectadas por Quiroga (2011), Gil Báez et al. (2015) y Tomas et al. (2012).

La autogamia presenta ventajas reproductivas que incluyen la fijación y mantenimiento de genotipos altamente adaptados al hábitat (Richards, 1986, Campbell et al., 1983) y el aseguramiento de una adecuada producción de frutos cuando los apareamientos son escasos (Inouye et al., 1996, Busch \& Delph, 2012) o cuando es ineficiente la transferencia de polen (Campbel et al., 1983). La distribución natural de estas especies, en particular T. crinita, incluye ambientes restrictivos (aridez y salinidad) por lo que la autogamia y su efecto (el aseguramiento reproductivo) sería parte de una estrategia dirigida a garantizar la reproducción.

La variación hallada en el peso de los frutos entre las poblaciones analizadas de ambas especies coincide con lo registrado por Zabala et al. (2011) para otros materiales del género. Las plantas producen frutos de distintos tamaños y con diversos grados de dormancia, lo cual les permite incrementar sus posibilidades de perpetuación, especialmente en el caso de especies que viven en ambientes poco estables (Egley, 1995, Leishman et al., 2000). En relación con esto, Zabala et al. (2011) hallaron, para ambas especies de Trichloris, una correlación positiva entre la germinación y el peso de los frutos a temperaturas sub-óptimas de germinación. En gramíneas, el mayor peso de los frutos se atribuye a un incremento en el endosperma, ya que el volumen del embrión es normalmente bajo (Kos \& Poschlod, 2010). Tanto en $T$. crinita como en $T$. pluriflora el origen del polen afectó el tamaño de los frutos; 
en ambos casos fueron más pesados los frutos producidos en polinización libre. Dos factores podrían explicar estas diferencias: la presencia del sobre y la competencia polínica. En las condiciones en las que se realizó el ensayo el primer factor no fue objetivamente evaluado ya que no se ensobraron espiguillas de la planta aislada; sin embargo, como se mencionó, se considera que el ensobrado no afectó significativamente la iniciación de frutos dada la elevada relación fr/fl obtenida. No obstante ello es probable que, al ensobrar las inflorescencias, algunas exigencias micrometeorológicas propias del llenado de frutos hayan sido subóptimas. Así, el mayor peso de los frutos producidos en condiciones de polinización libre podría deberse a una combinación de condiciones de llenado adecuadas y a un incremento en la disponibilidad de polen. El aumento en la cantidad de polen debió favorecer una mayor competencia polínica. Antecedentes sobre el efecto de la competencia polínica para el adecuado desarrollo de frutos fueron presentados, para diversas especies, por Schlichting et al. (1987), Winsor et al. (2000) y Rahme et al. (2009), entre otros.

Este trabajo presenta el primer antecedente sobre el sistema reproductivo de especies del género Trichloris, postulando que las mismas presentan reproducción sexual y son predominantemente autógamas. Dicha información resulta imprescindible para tareas de domesticación de estas valiosas forrajeras nativas. Por otra parte, la elevada relación fr/fl detectada garantiza superar una importante barrera que presentan numerosas especies de gramíneas forrajeras nativas, como es la baja producción de frutos, posibilitando su selección e introducción al cultivo.

\section{Agradecimientos}

A la Dra. Beatriz Galati quien corroboró las observaciones embriológicas.

Este estudio fue totalmente financiado con fondos de un Proyecto (CAI+D), proveniente de la Universidad Nacional del Litoral.

\section{Bibliografía}

ANTON, A. M. \&A. E. 1984. The grass megagametophyte and its possible phylogenetic implications. Plant Syst. Evol. 146: 117-121. doi:10.1007/BF00984058.

ARACNE, M. J. 2010. Efecto de la polinización libre y la autopolinización en el éxito reproductivo pre-emergente y la germinación en poblaciones de Sporobolus indicus (Poaceae). Tesis de grado. Facultad de Ciencias Agrarias, Univ. Nacional del Litoral. Argentina. 34 pp.

ARONSON, J. A. 1989. Haloph, a data base of salt tolerant plants of the world. Univ. of Arizona, Office of Arid land Studies, $77 \mathrm{pp}$.

BORDÓN, A. 1981. Recursos naturales del chaco árido y semiárido: aprovechamiento forrajero de especies leñosas y herbáceas. Documento $\mathrm{n}^{\circ} 1218$ del Departamento de Producción Animal. INTA EERA Sáenz Peña (Material mimeográfico).

BRAGADÍN, L. 1959. Las pasturas en la región de los Llanos, Argentina. Revista Agronómica del Noroeste Argentino 3: 289-334.

BUSCH, J. W. \& L. F. DELPH. 2012. The relative importance of reproductive assurance and automatic selection as hypotheses for the evolution of selffertilization. Ann. Bot.-London 109: 553-562.

CABRERA, A. L. 1994. Regiones fitogeográficas argentinas. En: ACME (eds.), Enciclopedia Argentina de Agricultura y Jardinería, pp. 1-85. Buenos Aires, Argentina.

CAMPBELL, C. S., J. A. QUINN, G. P. CHEPLICK \& T. J. BELL. 1983. Cleistogamy in grasses. Annu. Rev. Ecol. Syst. 14: 411-441.

CAVAGNARO, J. B. 1988. Distribution of C3 and C4 grasses at different altitudes in a temperature arid region of Argentina. Oecologia 76: 273-277.

CAVAGNARO, J. B. \& A. D. DALMASSO. 1983. Respuesta a la intensidad y frecuencia de corte en gramíneas nativas de Mendoza. I. Pappophorum caespitosum y Trichloris crinita. Deserta 7: 203208.

CAVAGNARO, P. F., J. B. CAVAGNARO, J. L. LEMES, R. W. MASUELLI \& C. B. PASSERA. 2006. Genetic diversity among varieties of the native forage grass Trichloris crinita based on AFLP markers, morphological characters, and quantitative agronomic traits. Genome 49: 906-918.

CERQueIRA, E. D., A. M. SÁEZ \& C. M. RABOTNIKOF. 2004. Seasonal nutritive value of native grasses of Argentina Calden Forest Range. $J$. Arid Environ. 59: 645-656.

CHARNOV, E. L. \& J. J. BULL. 1986. Sex allocation, pollinator attraction and fruit dispersal in cosexual plants. J. Theor. Biol. 118: 321-325.

COVAS, G. 1978. Forrajeras indígenas, especies que requieren un plan de conservación de germoplasma. Ciencia e Investigación 34: 209-213.

CRUDEN, R. W. 1977. Pollen-ovule ratios: A 


\section{H. F. Gutiérrez et al. - Sistema reproductivo de Trichloris}

conservative indicator of breeding systems in flowering plants. Evolution 31: 32-46.

D’AMBRogiO, A. 1986. Manual de técnicas en histología vegetal. Hemisferio Sur, Buenos Aires.

DELESALlE, V. A., S. J. MAZER \& H. PAZ. 2008. Temporal variation in the pollen:ovule ratios of Clarkia (Onagraceae) taxa with contrasting mating systems: field populations. J. Evolution. Biol. 21: 310-323.

DÍAZ, H. 1965a. Las pasturas naturales en la zona del Bachi (Dpto. Burruyacu, Tucumán). Revista Industrial y Agrícola de Tucumán 43.

DÍAZ, H. 1965b. Valor forrajero de las pasturas naturales de la zona de Rodeo Grande, Tucumán. Revista Agronómica del Noroeste Argentino 4.

EGLEY, G. H. 1995. Seed germination in soil: Dormancy cycles. En: Kigel, J. \& G. Galilli (Eds.) Seed development and germination, pp. 529-543. MarcelDekker, Inc., New York, USA.

FEHR, W. R. 1987. Principles of cultivar development: Theory and Technique. McGraw-Hill, Inc. New York, New York.

GIL BÁEZ, C., R. ORDINOLAAGÜERO, R. D. ERNST \& M. A. RUIZ. 2015. Caracterización morfológica, biomasa aérea y calidad en distintas poblaciones de Trichloris crinita. Arch. Zootec. 64 (245): 49-56.

GOODWILLIE, C., R. D. SARGENT, C. G. ECKERT, E. ELLE, M. A. GEBER, M. O. JOHNSTON, S. KALISZ, D. A. MOELlER, R. H. REE, M. VALLEJO-MARIN \& A. A. WINN. 2010. Correlated evolution of mating system and floral display traits in flowering plants and its implications for the distribution of mating system variation. New Phytol. 185: 311-321.

GRECO, S. A. \& J. B. CAVAGNARO. 2003. Effect of drought in biomass production and allocation in three varieties of Trichloris crinita (Poaceae) a forage grass from the arid Monte region of Argentina. Plant Ecol. 64(1): 125-135.

GRECO, S. A. \& J. B. CAVAGNARO. 2005. Growth characteristics associated with biomass production in three varieties of Trichloris crinita (Poaceae), a forage grass native to the arid regions of Argentina. Rangeland J. 27: 135-142.

GUTIÉRREZ, H. F., D. MEDAN \& J. F. PENSIERO. 2006. Factors limiting pre-emergent reproductive success of Bromus auleticus Trin. ex Nees (Poaceae). 2. Fruit production under different pollination regimes, pollen viability and incompatibility reactions. New Zeal J. Bot. 44(1): 57-63.

HERLIHY, C. R. \& C. G. ECKERT. 2007. Evolutionary analysis of a key floral trait in Aquilegia canadensis (Ranunculaceae): Genetic variation in herkogamy and its effect on the mating system. Evolution 61: 1661-1674.
INFOSTAT 2014. InfoStat versión 2014-p2. Software estadístico Grupo InfoStat, FCA, Universidad Nacional de Córdoba, Argentina.

INOUYE, K., M. MAKI \& M. MASUDA. 1996. Evolution of Campanula flowers in relation to insect pollinators on islands. En: Lloyd DG, Barrett SCH. eds. Floral biology: studies on floral evolution in animal-pollinated plants, pp. 277-301. New York, NY: Chapman and Hall.

JOHNSTON, M. R., A. OLIVARES \& J. LAURA. 2003. Producción y calidad de semillas en cuatro Poáceas. Efecto de cortes con distintas frecuencias y en diversas etapas fenológicas. Agr. Téc. (Chile) 63(2): 146-155.

JOHRI, B. M, K. B. AMBEGAOKAR \& P. SRIVASTAVA. 1992. Comparative embryology of angiosperms. Springer-Verlag, New York.

KALISZ, S, A. RANDLE, D. CHAIFFETZ, M. FAIGELES, A. BUTERA \& C. BEIGHT. 2012. Dichogamy, outcrossing rates and selfing syndrome in mixed-mating Collinsia. Ann. Bot.-London 109: 571-582. doi:10.1093/aob/mcr237.

KOS, M. \& P. POSCHLOD. 2010. Why wait? Trait and habitat correlates of variation in germination speed among Kalahari annuals. Oecologia 162: 549-559.

KozUB, C., P. F. CAVAGNARO, A. LÓPEZ, C. QUARIN, J. B. CAVAGNARO. 2011. Estudios sobre el método reproductivo de Trichloris crinita, una gramínea forrajera del monte argentino. Congreso; 2da Reunión Conjunta de Sociedades de Biología de la República Argentina; San Juan. Abstract pp. 13.

KUNST, C., R. RENOLFI, H. PÉREZ \& F. DALLA TEA. 1986. Clasificación de gramíneas de bosques y arbustales de la región chaqueña occidental por sus caracteres ecológicos y productivos. Revista Argentina de Producción Animal 6, Supl. 1: 6.

KUNST, C., F. DELLA TEA, H. PÉREZ \& R. RENOLFI. 1987. Vegetación natural y oferta de biomasa herbácea del sitio "Valle fluvial actual del Río Dulce", región chaqueña occidental, Santiago del Estero. Revista Argentina de Producción Animal 7: 163-179.

KUNST, C., R. RENOLFI, H. PÉREZ \& F. DALLA TEA. 1995. Preferencia de bovinos por gramíneas nativas de bosques y arbustales de la región chaqueña occidental. Revista Argentina de Producción Animal 15: $110-113$.

KUNST, C., R. LEDESMA, E. MONTI, J. CASILLO \& J. GODOY. 2007. Gramíneas indicadoras de condición en sitios de pastizal del sudoeste de Santiago del Estero. Rev. Investig. Agropecu. 36(1): 33-61

LEISHMAN, M. R., I. J. WRIGHT, A. T. MOLES \& M. WESTOBY. 2000. The evolutionary ecology of seed size. En: Fenner, M. (Ed.). Seeds - The Ecology of 
Regeneration in Plant Communities, pp 31-57, 2nd edition. CAB International, Wallingford.

LLOYD-REILLEY, J. \& E. KADIN. 2002. False rhodes grass, Chloris crinita Lag. Plant Fact Sheet. USDA, NRCS, Kika de la Garza Plant Material Center.

MAZER, S. J., L. S. DUDLEY, V. A. DELESALLE, H. PAZ \& P. GALUSKY. 2009. Stability of pollen-ovule ratios in pollinator-dependent versus autogamous Clarkia sister taxa: testing evolutionary predictions. New Phytol. 183: 630-648.

MAZER, S. J., L. S. DUDLEY, A. A. HOVE, S. K. EMMS \& A. S. VERHOEVEN. 2010. Physiological performance in Clarkia sister taxa with contrasting mating systems: do early-flowering autogamous taxa avoid water stress relative to their pollinatordependent counterparts? Int. J. Plant Sci. 171: 1029-1047.

MORELLO, J. \& C. SARAVIA TOLEDO. 1959. El bosque chaqueño I y II. Revista Agronómica del Noroeste Argentino 3: 5-81/209-258.

NICORA, E. G. \& Z. E. RÚGOLO. 1987. Los géneros de gramíneas de América Austral. Ed. Hemisferio Sur. Buenos Aires.

PENSIERO, J. F., H. F. GUTIÉRREZ \& E. EXNER. 2005. Sistema de polinización y su efecto sobre la producción y el peso de semillas en nueve especies sudamericanas del género Setaria. Interciencia 30(8): 495-500.

PETERSON, P. M. 2001. Trichloris. En Peterson, P. M., R. J. Soreng, G. Davidse, T. Filgueiras, F. O. Zuloaga \& E. J. Judwiewicz. Catalogue of New World Grasses (Poaceae): II. Subfamily Chloridoideae. Contributions from the United States National Herbarium 41: 222-223.

QUERO CARRILLO, A. R., J. F. ENRÍQUEZ QUIROZ, C. R. MORALES NIETO \& L. M. JIMÉNEZ. 2010. Apomixis y su importancia en la selección y mejoramiento de gramíneas forrajeras tropicales. Revisión. Rev. Mex. Cienc. Pecu. 1(1): 25-42.

QUIROGA, R. E. 2011. Variación morfológica en once poblaciones del pasto nativo Trichloris crinita. $34^{\circ}$ Congreso Argentino de Producción Animal. Joint Meeting ASAS-AAPA. Mar del Plata, Buenos Aires. Abstract pp. 539.

RAGONESE, A. E. 1956. Recuperación de zonas semiáridas degradadas y resiembra de pasturas naturales en Argentina. Revista de Información sobre Investigación y Desarrollo Agropecuario (IDIA) 97: 14-17.

RAHME, J., A. WIDMER \& S. KARRENBERG. 2009. Pollen competition as an asymmetric reproductive barrier between two closely related Silene species. $J$. Evolution Biol. 22: 1937-1943.
RICHARDS, A. J. 1986. Plant Breeding Systems. George Allen and Unwin Publisher, London.

RUNIONS, C. J. \& M. A. GEBER. 2000. Evolution of the self-pollinating flower in Clarkia xantiana (Onagraceae). I. Size and development of floral organs. Am. J. Bot. 87: 1439-1451.

RUZIN, S. E. 1999. Plant Microtechnique and Microscopy. Oxford University Press, New York.

SCHLICHTING, C. D., A. G. STEPHENSON \& L. E. DAVIS. 1987. Pollen competition and offspring variance. Evol. Trend Plant 1: 35-39.

TOMAS, P., F. VACCARI, J. ZABALA, J. GIAVEDONI \& J. F. PENSIERO. 2012. Análisis de la variación genética en accesiones nativas de Trichloris crinita y Trichloris pluriflora mediante marcadores moleculares ISSR. Jornadas Latinoamericanas de Recursos Genéticos, Mejoramiento y Biotecnología de Especies Forrajeras. Pergamino, Buenos Aires.

WAINSTEIN, P. \& S. GONZÁLEZ. 1971. Valor nutritivo de plantas forrajeras del este de la provincia de Mendoza (Reserva de Ñacunán) II. Deserta 2: 77-85.

WEBB, C. J. \& D. G. LLOYD. 1986. The avoidance of interference between the presentation of pollen and stigmas in angiosperms. II. Herkogamy. New Zeal. J. Bot. 24: 163-178.

WIENS, D., C. L. CALVIN, C. A. WILSON, C. I. DAVERN, D. FRANK \& S. R. SEAVEY. 1987. Reproductive success, spontaneous embryo abortion, and genetic load in flowering plants. Oecologia 71(4): 501-509.

WINSOR, J. A., S. PERETZ \& A. G. STEPHENSON. 2000. Pollen competition in a natural population of Cucurbita foetidissima (Cucurbitaceae). Am. J. Bot. 87(4): 527-532.

ZABALA, J. M., P. WIDENHORN \& J. F. PENSIERO. 2011. Germination pattern of species of the genus Trichloris in arid and semiarid environments. Seed Sci. Technol. 39: 338-353. DOI: http://dx.doi. org/10.15258/sst.2011.39.2.07.

ZULOAGA, F. O., E. G. NICORA, Z. E. RÚGOLO DE AGRASAR, O. MORRONE, J. F. PENSIERO \& A. M. CIALDELLA. 1994. Catálogo de la familia Poaceae en la República Argentina. Missouri Botanical Garden, Monographs Systematic Botany 47: 1-178.

Recibido el 27 de julio de 2015, aceptado el 03 de septiembre de 2015. 